\title{
Performance Analysis of Self-Scheduling Multi-channel Cognitive MAC Protocols under Imperfect Sensing Environment
}

\author{
Mingyu Lee ${ }^{1}$, Seyoun Lim², Tae-Jin Lee ${ }^{1 *}$ \\ ${ }^{1}$ College of Information and Communication Engineering, Sungkyunkwan University, Suwon, Korea. \\ 2 DMC R\&D Center, Samsung Electronics, Suwon, Korea. \\ * Corresponding author. Tel.: +82-31-290-7149; email: tjlee@skku.edu \\ Manuscript submitted October 10, 2014; accepted April 25, 2015. \\ doi: 10.17706/ijcce.2015.4.5.327-335
}

\begin{abstract}
Cognitive radio MAC protocols have been proposed to efficiently utilize radio resources under the assumption that radio spectrum can be perfectly sensed. However, due to dynamic and agile radio characteristics, secondary users may imperfectly detect whether the radio resources are occupied or not. In this paper, we study how the imperfect sensing environment affects cognitive radio systems. The sensing errors, i.e., misdetection and false alarm, result in interference to the primary users and decrease the transmission opportunities of secondary users. We formulate the number of sensed idle channels for different sensing mechanisms and the throughput of the multi-channel cognitive MAC protocol with the misdetection and the false alarm probabilities. Simulations are also conducted to verify the analysis. According to the results, the imperfect sensing should be considered as a critical factor when the cognitive MAC protocols are designed due to the influence on primary users' and secondary users' transmissions.
\end{abstract}

Key words: Cognitive radio, MAC protocols, ad-hoc networks, multi-channel, imperfect sensing.

\section{Introduction}

There are some CR MAC protocols in [1]-[6], whose sensing methods are implicitly based on energy detection. Those protocols have been proposed emphasizing how SUs can efficiently utilize the licensed spectrum without inference to PUs. Lim et al. in [1] propose a self-scheduling multi-channel cognitive radio MAC (SMC-MAC) protocol, which allows multiple SUs to transmit data simultaneously through the sensed idle channels by two cooperative channel sensing algorithms, i.e., fixed channel sensing (FCS) and adaptive channel sensing (ACS), and by slotted contention mechanism to exchange channel request information among SUs. Zhao et al. in [2] propose a decentralized cognitive radio MAC protocol based on the framework of partially observable Markov decision processes (POMDPs) to reduce the complexity of the optimal channel sensing and access. They assume multiple antennas to detect the status of licensed channels targeting the favorable sensing ability for the opportunistic frequency spectrum use. Su et al. in [3] and [4] propose the opportunistic multi-channel MAC protocols based on cross layer cooperation which integrates the spectrum sensing technique at the physical layer with the packet scheduling mechanism at the MAC layer for wireless ad-hoc networks. It requires two transceivers for control channel and data channels. A hardware-constrained cognitive MAC (HC-MAC) protocol [5] is proposed to reduce the computational complexity by approximating the optimal stopping rule for efficient spectrum sensing and access decision. 
The authors in [6] propose a MAC protocol based on a game-theoretic approach. It considers selfish cognitive radio devices with multiple radios and the simultaneous transmissions at different frequencies (channels) to efficiently coordinate and use the available channels.

Aforementioned MAC protocols assume that SUs can perfectly sense the radio spectrum. There, however, may be misdetection and the false alarm in sensing due to wireless radio characteristics. Wang et al. in [7] propose channel assignment algorithms: a heuristic and a greedy centralized scheme, using the information that SUs report to the secondary base station, to increase the number of available channels for multi-channel cognitive radio networks since the sensing performance in terms of misdetection and false alarm probabilities affects the overall system performance. Wong et al. in [8] design cognitive multi-channel MAC protocols with perfect and imperfect sensing, which enable SUs to opportunistically access available idle channels that a dedicated device reports to them.

In this paper, we consider the impact of imperfect sensing in a multi-channel cognitive radio ad-hoc network. We study the cognitive radio multi-channel MAC protocol in ad-hoc networks when sensing errors, i.e., the misdetection and false alarm, happen. The MAC protocol in [1] is considered as the multi-channel cognitive radio MAC and we study how channel sensing results by two sensing algorithms, i.e., FCS and ACS, are affected by misdetection and false alarm. Then we formulate the analytical model for the throughput of the multi-channel cognitive radio MAC protocol under imperfect sensing.

\section{System Model and MAC Protocol Description}

In this section, we describe the system model, imperfect sensing including both misdetection and false alarm and the CR multi-channel MAC protocol.

\subsection{System Model}

We consider a multi-channel system where primary users and $N_{s u}$ secondary users share radio resources under the imperfect sensing environment with two probabilities: misdetection and false alarm. For a primary user traffic model [1], all licensed channels have the same utilization $\alpha$ according to the PUs' traffic load, and there are $N_{c h}$ orthogonal channels. An SU network is a single hop cognitive radio network with $N_{s u}$ SUs under a primary user network. The SU network is an Ad-Hoc network which allows a node to communicate directly with another node within the transmission coverage.

\subsection{Imperfect Sensing}

We consider the imperfect sensing environment with the misdetection probability $p_{m d}$ and the false alarm probability $p_{f a}$. According to [8], the detection probability $p_{d e}$ and the false alarm probability $p_{f a}$ are considered under the energy detection mechanism. $p_{d e}$ is the probability that an occupied primary channel is declared to be correctly occupied and $p_{f a}$ is the probability that a vacant primary channel is declared to be occupied. So the misdetection probability $p_{m d}=1-p_{d e}$, the probability that an occupied primary channel is declared to be vacant. The misdetection and false alarm cause the interference to PUs and the lost transmission opportunity of SUs, respectively. In [8], the relationship between the sensing period of a channel, $T_{c y c l e}$, with the probability of false alarm, $p_{f a}$, for a given detection probability, $1-p_{m d}$, in the energy detector is given as follows.

$$
p_{f a}=Q\left(\sqrt{2 \gamma+1} \cdot Q^{-1}\left(1-p_{m d}\right)+\sqrt{T_{c y c l e}} \cdot \gamma\right)
$$

where $Q(\cdot)$ is the complementary distribution function of the standard Gaussian distribution, $\gamma$ is the received signal-to-noise ratio of the primary user measured at the secondary users's receiver of interest, 
under the hypothesis that the primary user is active, and $T_{c y c l e}$ is a cycle time.

\begin{tabular}{|c|c|c|c|}
\hline$\leftarrow T_{\text {idl } \vec{e} \rightarrow}$ & $T_{S S}=$ & $-T_{c F}$ & $-T_{t r}$ \\
\hline CR-idle & Sensing-Sharing & Contention & Transmission \\
\hline
\end{tabular}

Fig. 1. A cycle structure of CR multi-channel MAC protocol.

\subsection{MAC Protocol Description}

In the SMC-MAC protocol [1], SUs can use one common control channel and up to $N_{c h}$ primary channels for data transmission. A cycle time $\left(T_{c y c l e}\right)$ consists of four intervals: CR-Idle $\left(T_{\text {idle }}\right)$, Sensing-Sharing (SS) $\left(T_{s s}\right)$, Contention $\left(T_{c t}\right)$ and Transmission $\left(T_{t r}\right)$ as shown in Fig. 1. The CR-Idle interval is a constant time to indicate the beginning of a new cycle. The Sensing-Sharing interval, during which SUs sense the primary channels and exchange the sensing results on whether primary channels are idle or busy, is composed of $N_{c h}$ SS-slots each with three subslots. The first subslot is designed for sensing and the other two subslots are for sharing of the sensing result by tone signals. The contention interval consists of $M$ contention-slots, which enables SUs to compete with one another to reserve the sensed idle channels to be used to transmit their data frames during the transmission period. After contention, successful SUs can transmit data frames in parallel on self-scheduled idle channels during the transmission period. All SUs are assumed to be synchronized to cycle times, which is similar as in [5]. A new SU entering a secondary network listens to a control channel for the $T_{c t}$ ( $=M$ contention slots) contention interval to overhear CR-RTS and CR-CTS messages among other SUs in the network. The duration field in the messages indicates the data transmission time $\left(T_{t r}\right)$. This allows a new $\mathrm{SU}$ to know the end of data transmission in the current cycle. After the transmission time in the current cycle, the SU can synchronize to the next cycle.

\section{Analysis}

In this section, we analyze the performance of the multi-channel cognitive MAC protocol [1] under the imperfect sensing condition. The throughput of the MAC protocol under the imperfect sensing environment is formulated from the statistics of primary user channels [9], the number of sensed idle channels and the number of successful SUs.

We define the characteristics of the licensed channels as follows. The probability $p_{Y_{i}}\left(y_{i}\right)$ that the number of idle channels is $y_{i}$ among $N_{c h}$ licensed channels is

$$
p_{Y_{i}}\left(y_{i}\right)=\left(\begin{array}{c}
N_{c h} \\
y_{i}
\end{array}\right)(1-\alpha)^{y_{i}} \cdot \alpha^{N_{c h}-y_{i}}, 0 \leq y_{i} \leq N_{c h},
$$

where $\alpha$ is the primary users' traffic load. The probability $p_{Y_{b}}\left(y_{b}\right)$ that the number of busy channels is $y_{b}$ among $N_{c h}$ licensed channels.

$$
p_{Y_{b}}\left(y_{b}\right)=\left(\begin{array}{c}
N_{c h} \\
y_{b}
\end{array}\right) \alpha^{y_{b}} \cdot(1-\alpha)^{N_{c h}-y_{b}}, 0 \leq y_{b} \leq N_{c h} .
$$

We need to characterize the licensed channels that imperfect sensing affects. First of all, we define the probability that a channel state changes from idle (I) / busy (B) state to busy (B) /Idle (I) state by imperfect 
sensing. There are four types of the probabilities, $p_{I, I}\left(i_{n o f a}\right), p_{B, I}\left(i_{m d}\right), p_{I, B}\left(b_{f a}\right), p_{B, B}\left(b_{n o m d}\right)$ which are the probabilities with $i_{n o f a}$ idle channels without false alarm, $i_{m d}$ idle channels with miss detection, $b_{f a}$ busy channels with false alarm and $b_{\text {nomd }}$ busy channels without miss detection, respectively. Let $p_{f a}$ and $p_{m d}$ be the probability of false alarm and that of miss detection, respectively. The probability that there are $i_{\text {nofa }}$ idle channels without false alarm among $y_{i}$ idle channels channels is

$$
p_{I, I}\left(i_{\text {nofa }} \mid y_{i}\right)=\left(\begin{array}{c}
y_{i} \\
i_{\text {nofa }}
\end{array}\right)\left(1-p_{f a}\right)^{i_{n o f a}} \cdot p_{f a}^{y_{i}-i_{\text {nofa }}}, 0 \leq i_{\text {nofa }} \leq y_{i} \text {. }
$$

Then, the probability $p_{I, I}\left(i_{n o f a}\right)$ is given as follows,

$$
p_{I, I}\left(i_{\text {nofa }}\right)=\sum_{y_{i}=0}^{N_{c h}} p_{I, I}\left(i_{\text {nofa }} \mid y_{i}\right) \cdot p_{Y_{i}}\left(y_{i}\right), 0 \leq i_{\text {nofa }} \leq y_{i} .
$$

Let $I_{\text {nofa }}$ be the number of idle channels without false alarm. Then the average number of idle channels without false alarm is

$$
E\left[I_{\text {nofa }}\right]=\sum_{y_{i}=0}^{N_{c h}} \sum_{i_{\text {mofa }}=0}^{y_{i}} i_{n o f a} \cdot p_{I, I}\left(i_{\text {nofa }} \mid y_{i}\right) \cdot p_{Y_{i}}\left(y_{i}\right)
$$

Let $I_{m d}$ be the number of idle channels with misdetection. Then the probability that there are $i_{m d}$ busy channels but sensed as idle channels among $y_{b}$ busy channels by miss detection is

$$
p_{B, I}\left(i_{m d} \mid y_{b}\right)=\left(\begin{array}{l}
y_{b} \\
i_{m d}
\end{array}\right) p_{m d}^{i_{m d}} \cdot\left(1-p_{m d}\right)^{y_{b}-i_{m d}}, 0 \leq i_{m d} \leq y_{b} .
$$

From (7), $p_{B, I}\left(i_{m d}\right)$ is given as

$$
p_{B, I}\left(i_{m d}\right)=\sum_{y_{b}=0}^{N_{d h}} p_{B, I}\left(i_{m d} \mid y_{b}\right) \cdot p_{Y_{b}}\left(y_{b}\right)
$$

Then the average number of idle channels with misdetection is

$$
E\left[I_{m d}\right]=\sum_{y_{b}=0}^{N_{c h}} \sum_{i_{m d}=0}^{y_{b}} i_{m d} \cdot p_{B, I}\left(i_{m d} \mid y_{b}\right) \cdot p_{Y_{b}}\left(y_{b}\right)
$$

Under the imperfect sensing condition, we denote the channels that can be sensed as idle by $I^{\prime}$. They include $I_{\text {nofa }}$, idle channels without false alarm, and $I_{m d}$, idle channels with misdetection. Then the probability $p_{I^{\prime}}\left(i^{\prime}\right)$ that $i^{\prime}$ channels sensed as idle is

$$
p_{I^{\prime}}\left(i^{\prime}\right)=\sum_{i_{\text {ofs }}=0}^{i^{\prime}} p_{I, I}\left(i_{\text {nofa }}\right) \cdot p_{B, I}\left(i^{\prime}-i_{\text {nofa }}\right), 0 \leq i^{\prime} \leq N_{c h}
$$


From (5) and (7), the average number of sensed idle channels is

$$
E\left[I^{\prime}\right]=\sum_{i=0}^{N_{c h}} i^{\prime} \cdot p_{I}\left(i^{\prime}\right)=\sum_{i^{\prime}=0}^{N_{c h}} i^{\prime} \cdot \sum_{i_{\text {nofa }}=0}^{i^{\prime}} p_{I, I}\left(i_{n o f a}\right) \cdot p_{B, I}\left(i^{\prime}-i_{n o f a}\right) .
$$

\subsection{Channel Sensing}

We analyze the channel sensing mechanism in this section to formulate the average number of sensed idle channels under the imperfect sensing environment according to ACS [1]. According to the ACS algorithm [1], each SU senses $r$ channels $\left(C h_{\text {idle }} \leq r \leq C h_{\max }\right)$ randomly until it finds $x^{\prime}$ idle channels $\left(0 \leq x^{\prime} \leq C h_{\text {idle }}\right.$ ). Let $X^{\prime}$ be the number of sensed idle channels by an SU. Then the conditional probability $p_{X^{\prime}}\left(x^{\prime} \mid i^{\prime}\right)$ that the number of sensed idle channels $X^{\prime}=x^{\prime}$ given $i^{\prime}$ idle channels is as follows.

$$
p_{X^{\prime}}\left(x^{\prime} \mid i^{\prime}\right)=\left\{\begin{array}{c}
\left(\begin{array}{c}
C h_{\max } \\
x^{\prime}
\end{array}\right) \frac{\prod_{i=0}^{x^{\prime}-1}\left(i^{\prime}-i\right) \prod_{j=0}^{C h_{\max }-x^{\prime}-1}\left(N_{c h}-i^{\prime}-j\right)}{\prod_{k=0}^{C h_{\max }-1}\left(N_{c h}-k\right)}, 0 \leq x^{\prime}<C h_{\text {idle }} \\
\sum_{r=x^{\prime}}^{C h_{\max }}\left(\begin{array}{c}
r-1 \\
x^{\prime}-1
\end{array}\right) \frac{\prod_{i=0}^{x^{\prime}-1}\left(i^{\prime}-i\right) \prod_{j=0}^{r-x^{\prime}-1}\left(N_{c h}-i^{\prime}-j\right)}{\prod_{k=0}^{r-1}\left(N_{c h}-k\right)}, x^{\prime}=C h_{\text {idle }}
\end{array}\right.
$$

From (10) and (12), the average of $X^{\prime}$ sensed idle channels by an SU is

$$
E\left[X^{\prime}\right]=\sum_{i^{\prime}=0}^{N_{c h}} \sum_{x^{\prime}=0}^{i^{\prime}} x^{\prime} \cdot p_{X}\left(x^{\prime} \mid i^{\prime}\right) \cdot p_{I^{\prime}}\left(i^{\prime}\right)
$$

The probability $\gamma$ that an idle channel is sensed by an SU among the total idle channels is found as

$$
\gamma=\frac{\text { Average Number of Sensed Idle Channels by an SU }}{\text { Average Number of Idle Channels }}=\frac{E\left[X^{\prime}\right]}{E\left[I^{\prime}\right]}
$$

Since SUs independently select and sense the licensed channels, the probability $p_{U^{\prime}}\left(u^{\prime}\right)$ that an idle channel is sensed by $u^{\prime}$ SUs among $N_{s u}$ SUs is derived as

$$
p_{U^{\prime}}\left(u^{\prime}\right)=\left(\begin{array}{c}
N_{s u} \\
u^{\prime}
\end{array}\right) \gamma^{u^{\prime}} \cdot(1-\gamma)^{N_{s u}-u^{\prime}}, 0 \leq u^{\prime} \leq N_{s u}
$$

Then, the probability $\chi_{A C S}$ that an idle channel is sensed by at least one SU is derived as $\chi_{A C S}=1-(1-\gamma)^{N_{s u}}$. Let $W^{\prime}$ be the number of sensed idle channels by $N_{s u}$ SUs given total $I^{\prime}$ idle channels. Then the conditional probability $p_{W^{\prime}}\left(w^{\prime} \mid i^{\prime}\right)$ that $w^{\prime}$ idle channels are sensed by $N_{s u}$ SUs given $i^{\prime}$ idle channels following in (10) is derived as:

$$
p_{W^{\prime}}\left(w^{\prime} \mid i^{\prime}\right)=\left(\begin{array}{c}
i^{\prime} \\
w^{\prime}
\end{array}\right) \chi_{A C S}^{w^{\prime}} \cdot\left(1-\chi_{A C S}\right)^{i^{\prime}-w^{\prime}}, 0 \leq w^{\prime} \leq i^{\prime}
$$


So, the average number of sensed idle channels by $N_{s u}$ SUs is derived from (10) and (16).

$$
E\left[W^{\prime}\right]=\sum_{i^{\prime}=0}^{N_{c h}} \sum_{w^{\prime}=0}^{i^{\prime}} w^{\prime} \cdot p_{W^{\prime}}\left(w^{\prime} \mid i^{\prime}\right) \cdot p_{I^{\prime}}\left(i^{\prime}\right)
$$

\subsection{Contention}

Suppose that each SU randomly chooses a contention slot among $M$ slots in the contention interval. If a slot is selected by a single SU among $N_{s u}$ SUs, the slot is successful. Let $S$ be the number of successful slots and $p_{S}(s)$ be the probability that $s$ slots are successful, then

$$
p_{S}(s)=\left(\begin{array}{c}
M \\
s
\end{array}\right)\left(p_{\text {succ }}\right)^{s} \cdot\left(1-p_{\text {succ }}\right)^{M-s}, 0 \leq s \leq M
$$

where $\mathrm{p}_{\text {succ }}$ is the probability that a contention slot is selected by an SU and $p_{\text {succ }}=N_{s u} \cdot v \cdot(1-v)^{N_{s u}-1}$, $v=\frac{1}{M}[1]$. From (18), the average number of successful contention slots is

$$
E[S]=\sum_{s=0}^{M} s \cdot p_{S}(s)
$$

\subsection{Throughput}

We, first, derive the transmission time per data channel denoted by $T_{t r}$ (see Fig. 1) [1], $T_{t r}=T_{\text {cycle }}-\left(T_{\text {idle }}+T_{s s}+T_{c t}\right)$, where $T_{\text {cycle }}$ is the cycle time, $T_{i d l e}$ is the idle time, $T_{s s}$ is the sensing-sharing time, and $T_{c t}$ is the contention time. The throughput of total sensed idle channels with the ACS mechanism is denoted by $T h_{\text {total }}^{A C S}$ as follows.

$$
T h_{\text {total }}^{A C S}=\left(E\left[\min \left(S \cdot C h_{\text {idle }}, W^{\prime}\right)\right] \cdot T_{t r} \cdot R\right) / T_{\text {cycle }} .
$$

Let $Z_{A C S}^{\prime}$ be $Z_{A C S}^{\prime}=\min \left(S \cdot C h_{\text {idle }}, W^{\prime}\right)$, and the pmf of $Z_{A C S}^{\prime}$ is

$$
p_{Z_{A C S}^{\prime}}\left(z_{A C S}^{\prime}\right)=F_{Z_{A C S}^{\prime}}\left(z_{A C S}^{\prime}\right)-F_{Z_{A C S}^{\prime}}\left(z_{A C S}^{\prime}-1\right), 0<z_{A C S}^{\prime} \leq N_{c h}
$$

where $p_{Z_{A C S}^{\prime}}(0)=F_{Z_{A C S}^{\prime}}(0)$. The mean of $z_{A C S}^{\prime}$ is

$$
E\left[Z_{A C S}^{\prime}\right]=\sum_{z_{A C S}^{\prime}=0}^{N_{c h}} z_{A C S}^{\prime} \cdot p_{Z_{A C S}^{\prime}}\left(z_{A C S}^{\prime}\right)
$$

We also need to find the throughput of sensed idle channels affected by misdetection. The probability that the number of idle channels affected by misdetection is $Z_{m d}^{A C S}$ among $Z_{A C S}^{\prime}$ is

$$
p_{Z_{m d}^{A C S}}\left(z_{m d}^{A C S} \mid z_{A C S}^{\prime}\right)=\left(\begin{array}{l}
z_{A C S}^{\prime} \\
z_{m d}^{A C S}
\end{array}\right) v^{z_{m d}^{A C S}} \cdot(1-v)^{z_{A C S}^{\prime}-z_{m d}^{A C S}}, 0 \leq z_{m d}^{A C S} \leq z_{A C S}^{\prime},
$$


where $v=\frac{E\left[I_{m d}\right]}{E\left[I_{I^{\prime}}\right]}$ from (9) and (11). The mean of $Z_{m d}^{A C S}$ is as follows,

$$
E\left[Z_{m d}^{A C S}\right]=\sum_{z_{A C S}^{\prime}=0}^{N_{c h}} \sum_{z_{m d}^{A C S}=0}^{z_{A C S}^{\prime}} z_{m d}^{A C S} \cdot p_{Z_{m d}^{A C S}}\left(z_{m d}^{A C S} \mid z_{A C S}^{\prime}\right) \cdot p_{Z_{A c S}^{\prime}}\left(z_{A C S}^{\prime}\right)
$$

Then, the throughput by misdetection is

$$
T h_{m d}^{A C S}=\left(E\left[Z_{m d}^{A C S}\right] \cdot T_{t r} \cdot R\right) / T_{c y c l e} .
$$

Using (20) and (25), we can obtain the actual throughput of ACS without misdetection

$$
T h_{\text {real }}^{A C S}=T h_{\text {total }}^{A C S}-T h_{m d}^{A C S} .
$$

\section{Simulation}

In this section, we present simulation results and the performance of the cognitive MAC protocol under the imperfect sensing environment. We also verify the analysis with the simulation. Table 1 summarizes the parameters for an SU network under the imperfect sensing environment to evaluate the performance of the cognitive multichannel MAC protocol. The basic parameters for an SU network are employed from IEEE 802.11a [10]. $T_{i d l e}$ is given as aSIFSTime $+2 \times$ aSlotTime and $T_{s S}$ is $N_{c h} \times$ aSlotTime $(=3 \times$ aSlotTime). $T_{c t}$ is $M \times$ aSlotTime. A contention slot time consists of CR-RTS transmission time, CR-SIFS and CR-CTS transmission time. When the CR-RTS and CR-CTS are transmitted, they are converted to the physical layer convergence procedure (PLCP) protocol data units (PPDUs) including a PLCP preamble and a PLCP header. So, the transmission time of CR-RTS/CR-CTS is 24s, and CR-SIFS is $16 \mu s$ (=aSIFSTime).

Table 1. Simulation Parameters

\begin{tabular}{cccc}
\hline \hline Parameter & Value & Parameter & Value \\
\hline Main channel Simulation Time & $1,000,000$ cycles & Number of Contention Slots $(M)$ & 10 slots \\
Assistant channel Data Rate $(\mathrm{R})$ & $54 \mathrm{Mbps}$ & Number of SUs $\left(N_{s u}\right)$ & $2-20 \mathrm{SUs}$ \\
$N_{c h}$ & 20 channels & CR-SIFS Time (aSIFSTime) & $16 \mu \mathrm{s}$ \\
Traffic load of PUs $(\alpha)$ & $0.0-1.0$ & Slot Time (aSlotTime) & $9 \mu \mathrm{s}$ \\
$C h_{\max }$ & $2-5$ channels & PLCP Preamble & $16 \mu \mathrm{s}$ \\
$C h_{\text {idle }}$ & 2 channels & PLCP Header & $4 \mu \mathrm{s}$ \\
Misdetection Probability $\left(p_{m d}\right)$ & $0.1-0.3$ & CR-RTS & $24 \mu \mathrm{s}$ \\
False Alarm Probability $\left(p_{f a}\right)$ & 0.1 & CR-CTS & $24 \mu \mathrm{s}$ \\
\hline \hline
\end{tabular}

Fig. 2(a) shows the analysis and simulation results of the multi-channel cognitive MAC protocol under the imperfect sensing environment when $p_{m d}=0.1, p_{f a}=0.1, M=10, T_{\text {cycle }}=1 s, C h_{\text {idle }}=2$ and $C h_{\text {max }}=5$, and they are compared with those under the perfect sensing environment. The throughputs of FCS are greater than those of ACS because an SU in FCS senses more channels. The throughputs under the imperfect sensing condition is lower than those under the perfect sensing condition since SUs lose the transmission opportunities due to the affected idle channels by misdetection. For example, when $\alpha=0.4$, the real throughputs of FCS and ACS under the imperfect sensing condition are $365.39 \mathrm{Mbps}$ and352.01 Mbps, respectively, and those of FCS and ACS under the perfect sensing condition are 394.83 Mbps and $380.20 \mathrm{Mbps}$, respectively. The throughputs of ACS for analysis and simulation are closely matched with each other as indicated in Fig. 2(a), which verifies our analysis. 


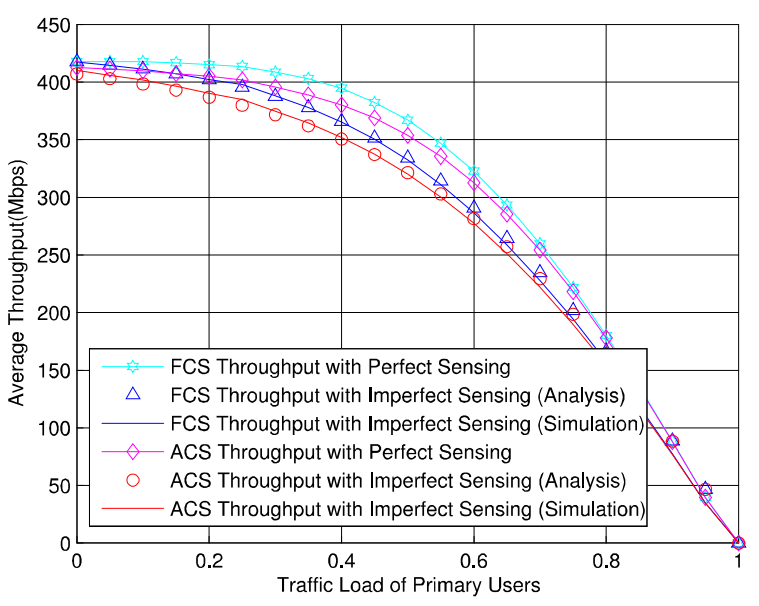

(a)

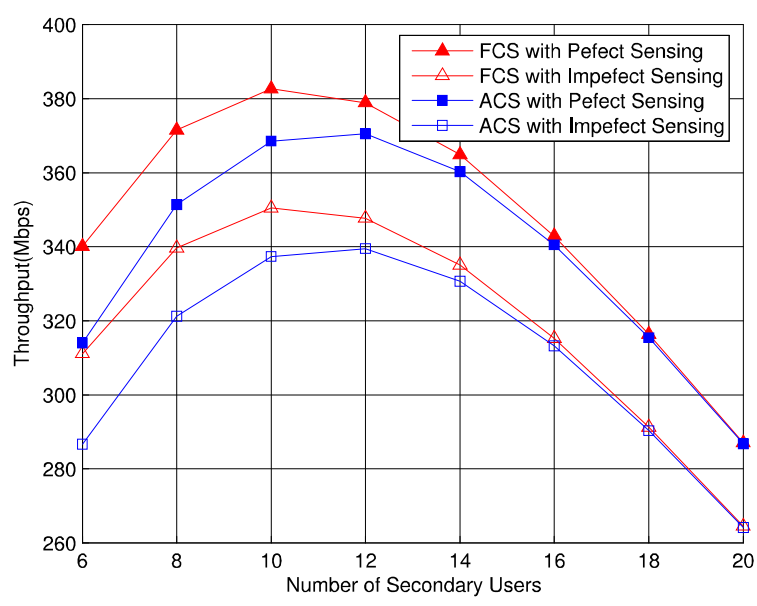

(b)

Fig. 2. Comparison of the average throughputs in the perfect sensing and the imperfect sensing environment.

The impact of the size of the contention slots $M$ on the throughputs is shown in Fig. 2(b) As the number of SUs varies from 6 to 20, the average throughputs are presented with parameters: $\alpha=0.5, M=10$, $T_{\text {cycle }}=1 \mathrm{~s}, C h_{\text {idle }}=2, C h_{\max }=5, p_{m d}=0.1$ and $p_{f a}=0.1$. The throughputs are shown to be maximized when $\mathrm{N}_{\mathrm{SU}}=\mathrm{M}$ and then to start to decrease around $N_{S U}=M$ since the limited number of contention slots causes more collisions. For instance, the maximum throughputs of FCS and ACS with imperfect sensing are 350.5 Mbps and 339.52 Mbps at $N_{S U}=10$, and then decrease to 291.29 Mbps and 290.38 Mbps, at $N_{S U}=18$, respectively. Besides, the throughputs with the imperfect sensing condition are compared with those with the perfect sensing case. The former is lower than the latter since imperfect sensing causes the interference to PUs and the loss of the SUs' access opportunities to the available licensed channels.

\section{Conclusion}

In this paper, we have considered the multi-channel cognitive MAC protocols under the imperfect sensing environment with misdetection and false alarm relaxing the tight assumption that channels are sensed perfectly without errors. The throughput of the MAC protocol with imperfect sensing is formulated in terms of the number of sensed idle channels considering the false alarm probability and the misdetection probability which may cause the interference to the PUs and decrease the transmission opportunities of SUs. We then have evaluated the performance of the protocol by extensive simulations. From the evaluation, the imperfect sensing conditions such as misdetection and false alarm need to be considered as a critical factor when CR MAC protocols are designed to reduce interference to PUs and to improve the performance of SUs.

\section{Acknowledgment}

This work was supported by the National Research Foundation of Korea (NRF) grant funded by the Korean government (MSIP) (2014R1A5A1011478).

\section{References}

[1] Lim, S., \& Lee, T.-J. (2011). A self-scheduling multi-channel cognitive radio MAC protocol based on cooperative communications. IEICE Trans. on Commun., E94-B(6), 1657-1668.

[2] Zhao, Q., Tong, L., Swami, A., \& Chen, Y. (2007). Decentralized cognitive MAC for opportunistic spectrum access in Ad-Hoc networks: A POMDP framework. IEEE J. Select. Areas Commun., 25(3), 589-600.

[3] Su, H., \& Zhang, X. (2007). Opportunistic MAC protocols for cognitive radio based wireless networks. 
Proceedings of CISS (pp. 363-368). Baltimore, Maryland, USA.

[4] Su, H., \& Zhang, X. (2008). Cross-layer based opportunistic MAC protocols for QoS provisioning over cognitive radio wireless networks. IEEE J. Select. Areas Commun., 26(1), 118-129.

[5] Jia, J., Zhang, Q., \& Shen, X. (2008). HC-MAC: A hardware-constrained cognitive MAC for efficient spectrum management. IEEE J. Select. Areas Commun., 26(1), 106-117.

[6] Felegyhazi, M., Cagalj, M., \& Hubaux, J. (2009). Efficient MAC in cognitive radio systems: A game-theoretic approach. IEEE Trans. Wireless Commun., 8(4), 1984-1995.

[7] Wang, W., Kasiri, B., Choi, J., \& Alfa, A. S. (2011). Channel assignment of cooperative spectrum sensing in multi-channel cognitive radio networks. Proceedings of IEEE ICC (pp. 1-5). Kyoto, Japan.

[8] Wong, D. T. C., Zheng, S., \& Liang, Y. (2011). Cognitive multi-channel MAC protocols with perfect and imperfect sensing. Proceedings of IEEE ICC (pp. 1-5). Kyoto, Japan.

[9] Willkomm, D., Machiraju, S., Bolot, J., \& Wolisz, A. (2009). Primary user behavior in cellular networks and implications for dynamic spectrum access. IEEE Commun. Mag., 47(3), 88-95.

[10] IEEE 802.11. (2007). Wireless LAN Medium Access Control (MAC) and Physical Layer (PHY) Specifications.

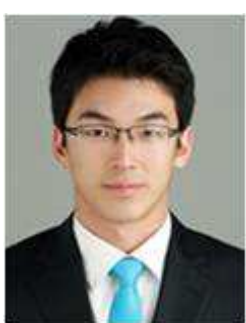

Mingyu Lee received the B.S. degree in electronics engineering from Kwangwoon University, Seoul, Korea in 2009 and the M.S. degree in mobile systems engineering from Sungkyunkwan University, Suwon, Korea in 2012. He is currently pursuing his Ph.D. degree in the Department of IT convergence at Sungkyunkwan University since March 2012. His research interests include cognitive networks, wireless LAN, and Ad-Hoc networks.

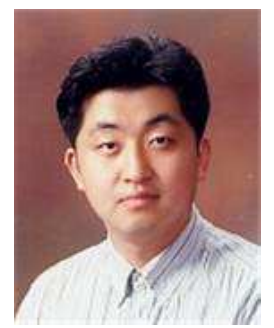

Seyoun Lim received the B.S and M.S. degrees in telecommunication \& information engineering from Korea Aerospace University, Goyang, Korea in 2000 and 2002, respectively and his Ph.D. degree in mobile systems engineering from Sungkyunkwan University, Suwon, Korea, in 2013. In 2002, he joined the Telecommunication R\&D Center in Samsung Electronics. He is working as a senior engineer at DMC R\&D Center in Samsung Electronics. His research interests include medium access control, resource allocation for cognitive radio networks.

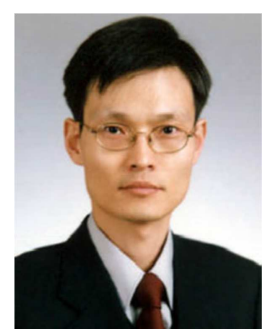

Tae-Jin Lee received his B.S. and M.S. degrees in electronics engineering from Yonsei University, Korea in 1989 and 1991, respectively, and the M.S.E. degree in electrical engineering and computer science from University of Michigan, Ann Arbor, in 1995. He received the Ph.D. degree in electrical and computer engineering from the University of Texas, Austin, in May 1999. In 1999, he joined the Corporate R \& D Center, Samsung Electronics where he was a senior engineer. Since 2001, he has been a professor in the College of Information and Communication Engineering at Sungkyunkwan University, Korea. He was a visiting professor in Pennsylvania State University from 2007 to 2008. His research interests include performance evaluation, resource allocation, medium access control (MAC), and design of communication networks and systems, wireless LAN/PAN/MAN, Ad-Hoc/sensor/RFID networks, next generation wireless communication systems. He has been a voting member of IEEE 802.11 WLAN Working Group, and is a member of IEEE and IEICE. 